\title{
A REFINEMENT OF THEOREMS OF KIRCHBERGER AND CARATHÉODORY
}

\author{
DONALD WATSON
}

(Received 19 May 1971; revised 8 July 1971)

Communicated by G. Szekeres

\section{Introduction}

I can indicate the type of refinement mentioned in the title by referring to Kirchberger's theorem [4]. Its picturesque form in the plane is: if sheep and goats are grazing in a field and for every four animals there exists a line separating the sheep from the goats then there exists such a line for all the animals. The refinement is that the words 'every four animals' may be replaced by 'every four animals including an arbitrarily chosen animal'; this reduces the 'Kirchberger number' from four to, effectively, three.

The starting point will be Helly's theorem [3] in $d$-dimensional Euclidean space $E_{d}$. Then follow refinements of the following chain of theorems: Helly's theorem in the surface $S_{d}$ of a sphere in $E_{d+1}$, Kirchberger's theorem in $S_{d}$ and in $E_{d}$, Carathéodory's theorem in $E_{d}$. For convenience of exposition the original name is retained, in coded form, for the refined version of a theorem. In each case the original form may be restored by deleting the reference to an arbitrarily chosen point.

In what follows convexity in $S_{d}$ will mean strong convexity; that is, a set in $S_{d}$ is called convex if it contains no pair of diametrically opposite points, and together with any two of its points contains the whole of the minor arc of the great circle containing them.

\section{Helly's Theorems in $S_{d}$}

First let us state Helly's theorem [3] in $E_{d}$ :-

THEOREM $\mathrm{HE}_{d}$. If every $d+1$ members of a finite family of convex sets in $E_{\mathrm{d}}$ have a common point then all menbers of the family have a common point.

We can readily deduce from this a refinement of Helly's theorem in $S_{d}$ :

THEOREM $\mathrm{HS}_{d}$. Let $F$ be a finite family of convex sets in $S_{d}$; if every $d+2$ 
of the sets, including an arbritarily chosen set, have a common point then all sets of $F$ have a common point.

To prove this let $C$ be the arbitrarily chosen set and consider the family $H$ of intersections of members of $F$ with $C$. $H$ will have the property that every $d$ +1 of its members have a common point. Now project $C$, from the centre of $S_{d}$, onto a hyperplane in $E_{d+1}$. We see, by theorem $\mathrm{HE}_{d}$ that all members of the projections of $H$ have a common point; hence all members of $H$ have a common point. It follows that all members of $F$ have a common point.

An immediate corollary to theorem $\mathrm{HS}_{d}$ is obtained by specifying $F$ to be a finite family of open hemi-spheres. It is a simple step then to the dual of theorem $\mathrm{HS}_{d}$; in replacing an open hemisphere by its antipodal pole, and vice versa, properties of incidence are preserved and theorem $\mathrm{HS}_{d}$ becomes

THEOREM DHS $\mathrm{d}_{\mathrm{d}}$ : Let $F$ be a finite family of points in $S_{\mathrm{d}}$, if every $d+2$ points, including an arbitrarily chosen one, lie in some open hemisphere, then all points of $F$ lie in an open hemisphere.

\section{Kirchberger's Theorem}

A simple proof of Kirchberger's theorem was given by Baker [1]; the same method can be used to derive the refinement:-

THEOREM $\mathrm{KS}_{d}$. Let $S$ and $G$ be disjoint finite sets of points in $S_{d}$; and let $p$ be an arbitrarily chosen point in the union $A$ of $S$ and $G$. If for each set $K$ satisfying

$$
|K|=d+2, \quad \quad \quad \quad K \in K, \quad K \subset A
$$

there exists a hyperplane through the centre stricly separating $K \cap S$ from $K \cap G$, then there exists a hyperplane through the centre stricly separating $S$ from $G$.

To prove this we reflect each point of $G$ through the centre into its antipodal point. Call the set of such points $G^{\prime}$. Then $S \cup G^{\prime}$ satisfy the conditions placed on $F$ in theorem $D H S_{d}$, hence the members of $S \cup G^{\prime}$ lie in some open hemisphere. A reflection of $G^{\prime}$ back onto $G$ yields the theorem.

An immediate consequence of the preceding theorem is:-

THEOREM $\mathrm{KE}_{d}$. Let $S$ and $G$ be disjoint finite sets of points in $E_{d}$; and let $p$ be any point in the union $A$ of $S$ and $G$. If for each set $K$ satisfying

$$
|K|=d+2, \quad \quad p \in K, \quad K \subset A
$$

there exists a hyperplane strictly separating $K \cap S$ from $K \cap G$, then there exists a hyperplane strictly separating $S$ from $G$. 
The proof is effected by embedding $E_{d}$ in $E_{d+1}$ and projecting $E_{d}$ onto an open hemisphere of $S_{d}$ by projecting through the centre of $S_{d}$. Theorem $K E_{d}$ then follows from theorem $K S_{d}$.

\section{Carathéodory's Theorem}

To obtain the refinement of Carathéodory's theorem [2] we express theorem $K E_{d}$ in contrapositive form and specialise it by taking $|S|=1, p \in G$. In $E_{d}$ the statement that a point can not be strictly separated by a hyperplane from a set of points is equivalent to the statement that the point is in the convex hull of the set. Hence we have:-

THEOREM $\mathrm{CE}_{d}$. If in $E_{d}$ a point is in the convex hull of a finite set of points $G$, where $|G|>d+1$, then it is in the convex hull of some $d+1$ points of $G$ including an arbitrarily chosen point of $G$.

\section{Acknowledgement}

I am grateful to Professor B.C. Rennie, Dr. M. J.C. Baker, and a referee for helpful criticism during the preparation of this paper.

\section{References}

[1] M. J. C. Baker, Families of interesting convex sets (Ph. D. Thesis, University of Melbourne, 1968.)

[2] C. Carathéodory, 'Über den Variabilitätsbereich der Koeffizienten von Potenzreihen, die gegebene Werte nicht annehmen', Math. Ann. 64 (1907), 95-115.

[3] E. Helly, 'Über Mengen konvexen Körper mit gemeinschaftlichen Punkten', Jber. Deutsch. Math. Verein. 32 (1923), 175-176.

[4] P. Kirchberger, 'Über Tschebyschefsche Annäherungsmethoden', Math. Ann. 57 (1903), 509-540.

Department of Mathematics

RAAF Academy Point Cook

Victoria, 3029

Australia 